\title{
Secuenciación de contenidos y objetos de aprendizaje
}

\section{Content sequencing and learning objects}

\author{
Miguel Zapata Ros \\ Universidad de Murcia, Murcia, España \\ mzapata@um.es
}

\begin{abstract}
Resumen
La selección y secuenciación de contenidos de enseñanza en el contexto de la planificación curricular y del pensamiento constructivista. La importancia de contar en el e-learning con herramientas y criterios autónomos que guíen el proceso de planificación y desarrollo desde unas bases propias, distintas de las derivadas de la configuración de la tecnología, o condicionado a la necesidad de contar con estándares de formato de intercambio de datos. Este planteamiento adquiere especial relevancia en el contexto del e-learning de propósito general, tanto en el de formación como en el e-learning empresarial o en el universitario. También señalamos las necesidades que plantea la industria del e-learning en la actualidad en relación con el diseño instruccional de objetos de aprendizaje, y que constituyen una prioridad y un desafío.

Fundamentación de las teorías que tratan la selección de contenidos. Presupuestos básicos y descripción de las técnicas de secuenciación. Nos centraremos en tres de ellas: Técnica de análisis de contenidos, técnica de análisis de la tarea y teoría de la elaboración.

Como conclusión intentaremos abordar, sólo en su propuesta como enunciado, dos cuestiones: Si el concepto de objeto de aprendizaje reutilizable es compatible con los requisitos de interdependencia de contenidos de aprendizaje. Y si es así ¿qué requisitos han de cumplir éstos?
\end{abstract}

Palabras clave: Objetos de aprendizaje, reusabilidad, usabilidad, estándares de calidad de aprendizaje en redes, e-learning, Teoría del Curriculum, secuenciación de contenidos de aprendizaje, Técnica de Análisis de Contenidos, Técnica de Análisis de la Tarea, Teoría de la Elaboración.

\begin{abstract}
This article presents in the first place a standpoint of content sequencing and selecting in curricular design, according to the constructivist view. In open and distance learning supported by networks, due to the urgent need to have data exchange format standards, it is very important to have tools and criteria curricularly based guiding this process instead of those coming from the technological tools used.

This approach is very much needed in e-learning in general, but also in corporate elearning and in e-learning in higher education.

In the second place, this article stresses the need, articulated by the business of elearning, of learning objects instructional design, and considers it as a priority and a challenge.

Thirdly, we review the theories and procedures of content sequencing, their basic assumptions, and the sequencing techniques, in particular Content Analysis Technique,

Task Analysis Technique, and Elaboration Theory.

Finally, as a conclusion, we will address several questions: The reusable learning object concept is it compatible with the interdependence requisites of learning contents? And if so, which are the requisites that the RLOs must meet?
\end{abstract}

Keywords: Learning objects, reusability, usability, learning technology standards, learning, Curriculum Theory, content sequencing, Content Analysis Technique, Task Analysis Technique, Elaboration Theory. 


\section{Introducción}

En este artículo planteamos una visión de la selección y secuenciación de contenidos de enseñanza, en el contexto de la planificación curricular, desde la perspectiva de las corrientes del pensamiento constructivista. Señalamos la importancia de contar, en el campo de la formación apoyada en redes, con herramientas y criterios autónomos que guíen este proceso desde unas bases propias, externas, en vez de las derivadas de la configuración de las herramientas tecnológicas, y desde la necesidad de contar con estándares de formato de intercambio de datos.

$\mathrm{Si}$, en general, este planteamiento es importante adquiere especial relevancia en el contexto del e-learning de propósito general, tanto en el de formación como en el elearning empresarial o en el universitario. Y por supuesto en el contexto de la formación reglada y de formación informal, o de la no reglada. También señalamos las necesidades que plantea la industria del e-learning en la actualidad en relación con el diseño instruccional de objetos de aprendizaje, necesidades que constituyen una prioridad y un desafío.

A continuación pasamos a desarrollar la fundamentación de las teorías que rigen los procedimientos de selección de contenidos, los presupuestos básicos y la descripción de las técnicas de secuenciación. En particular nos centraremos en tres de ellas: La técnica de análisis de contenidos, la técnica de análisis de la tarea y la teoría de la elaboración.

Por último como conclusión intentaremos abordar, no en su resolución sino sólo en su propuesta como enunciado, varias cuestiones: ¿el concepto de objeto de aprendizaje reutilizable es compatible con los requisitos de interdependencia de contenidos de aprendizaje? Y si es así ¿qué requisitos han de cumplir éstos?

1

\subsection{Los medios tecnológicos (redes y plataformas) como recursos formativos.}

\section{Entornos tecnológicos como entornos singulares de aprendizaje. La perspectiva constructivista y el diseño curricular}

Un punto de vista ya clásico, vigente en buena parte de la cultura del aprendizaje en los países de nuestro entorno, fundamentado en las teorías constructivistas del aprendizaje (las que hacen especial énfasis en la idea del alumno como centro de los procesos cognitivos y por ende de los procesos de enseñanza y de aprendizaje), es el que sitúa los recursos tecnológicos (ordenadores, redes, plataformas y programas) en un esquema complejo de contenidos, procesos y condiciones de aprendizaje, y de relaciones humanas.

Estos puntos de vista nos son especialmente familiares por la frecuencia con que los hemos utilizados y tenido en cuenta a la hora del diseño de actividades educativas, de la intervención formativa y de la acción de asesoramiento y difusión en cursos, jornadas, seminarios y otras actividades de perfeccionamiento. Lo que señalaremos a continuación es pues una síntesis que sólo pretende poner en situación lo que constituye el nudo de este trabajo y por tanto carece de la precisión conceptual deseable, pero que por razones compresibles no podemos desarrollar aquí. 
Desde la perspectiva del constructivismo (Gagné, 1971) en toda situación de aprendizaje hay presentes tres elementos, o grupos de elementos, claramente diferenciados: Los resultados del aprendizaje o contenidos (QUÉ se aprende), los procesos (CÓMO se aprende) y las condiciones de aprendizaje (lo que ha de cumplir una actividad o una situación para que el aprendizaje se produzca).

En esta línea de pensamiento los contenidos serían el resultado del aprendizaje, es decir el cambio que se produce en el material cognitivo del alumno entre el antes y el después de la actividad de aprendizaje (cambio entendido como incorporación de nuevo material, desecho del antiguo o cambio en el tipo de relaciones entre elementos de conocimiento y/o la forma de procesarlo). Los procesos serían el CÓMO se aprende, es decir la actividad cognitiva que se pone en marcha, o el aprendiz pone en marcha, para efectuar el aprendizaje (estrategias y estilos cognitivos) y que varían según el tipo de aprendizaje (según la naturaleza del contenido ---hechos, conceptos,... o del dominio disciplinar---) y según la información previa (ideas previas, experiencias). Pero en cualquier caso (Pozo, 1990) estos procesos tienen una característica común: son procesos propios, internos e inherentes al aprendiz, y en consecuencia sólo observables en sus efectos. En consecuencia la intervención del profesor para propiciar el cambio en el material cognitivo del que hemos hablado (del cambio conceptual), o dicho de otra forma los procesos de enseñanza, sólo puede intervenir para crear condiciones favorables a ese cambio. O para que el proceso de aprendizaje se desencadene y se desarrolle, dentro de unas condiciones favorables.

Cada aprendizaje requiere unas condiciones concretas y diferentes a otro. Condiciones a determinar mediante procesos de planificación y de evaluación. Estas condiciones de aprendizaje están determinadas por dos elementos: LOS RECURSOS EDUCATIVOS y las estrategias de enseñanza, y por la interacción de ambos. Es en este marco de referencia en el que entendemos el uso de los medios digitales, como recursos de formación (o de educación) específicos que favorecen el aprendizaje de ciertos contenidos (en particular de ciertos procedimientos, conceptos y destrezas) asociados a situaciones específicas de aprendizaje y en relación con estrategias didácticas propias.

Esta línea de pensamiento no es original ni nueva, corresponde a una adaptación particular de lo desarrollado en los albores de la Informática Educativa por Seymour Papert $^{1}$ (Papert, S. 1982) y por Horacio Reggini (Reggini, 1983), en menor medida, seguidores a su vez de Piaget y de la Escuela de Ginebra, y sus trabajos sobre la Psicología del Desarrollo.

Por otra parte Rodríguez-Roselló (1988) señala, refiriéndose a los ordenadores como medios didácticos, la singularidad de los contenidos que se pueden alcanzar con ellos y de las concepciones metodológicas subyacentes. Singularidad que fácilmente se puede referir también a las redes o a Internet: Capacidad de interacción, favorecedores de entornos de aprendizaje autónomo y de entornos abiertos. Y favorecedores de estrategias de exploración y descubrimiento.

A esta dimensión hay que añadir otra en la línea del desarrollo cultural señalado por Vygotski (1984) sobre la creación de instrumentos que amplían las capacidades humanas de conocer y aprender: "instrumentos que la especie humana ha elaborado en

\footnotetext{
${ }^{1}$ Ver http://www.papert.org/ y http://web.media.mit.edu/ papert/ y la web del Media Laboratory en el MIT http://www.media.mit.edu/people/bio_papert.html.
} 
el transcurso de las relaciones e intercambios sociales de sus miembros". Aplicable a los medios tecnológicos por cuanto encierran de capacidad de codificación y de representación simbólica y conceptual. En particular aplicable al ordenador como instrumento regulador de relación e intercambio (mediación), como señala Cole (1991, pág. 412, a través de Crook 1994 y 1998 págs 49-58).

Más recientemente se han incorporado otras perspectivas bien con planteamientos originales o bien como adaptación del pensamiento de los constructivistas o de Vygotski, nos referimos a la Teoría de la Conversación y a la Teoría del Aprendizaje Situado.

La Teoría de la Conversación (Pask, 1964) parte del punto de vista de Vygotsky (1978), de que el hecho de aprender es por naturaleza un fenómeno social, y aporta que la adquisición de nuevo conocimiento es el resultado de la interacción de individuos que participan en un diálogo y que aprender es un proceso dialéctico en el que un individuo contrasta, confronta, su punto de vista personal con el de otro hasta llegar a un acuerdo. En el transcurso de lo cual y como resultado se produce la incorporación del nuevo material cognitivo.

Desde este punto de vista Internet es un entorno que presupone una naturaleza social específica, la de individuos, grupos, etc. comunicados a través de la red o con su mediación, y también entraña un proceso a través del cual los aprendices crean una zona virtual de proximal development (Vygotsky, 1978): La red aumenta lo que el alumno es capaz de aprender con el concurso de los demás.

Hay otra teoría a la que se acude para defender la fiabilidad de Internet como medio de aprendizaje, es la Teoría del Conocimiento Situado (Young, 1993). De acuerdo con ella, el conocimiento es una relación activa entre el individuo y un determinado entorno, además el aprendizaje se produce cuando el aprendiz está envuelto activamente en un contexto instruccional de naturaleza compleja y real.

El entorno Internet responde a las premisas del conocimiento situado en sus dos características principales: realismo y complejidad.

Por un lado, Internet posibilita intercambios auténticos entre usuarios provenientes de contextos culturales diferentes pero con intereses similares (Brown, Collins y Duguid, 1989). Por otro lado, la naturaleza inestable del entorno Internet constituye una dificultad para los no iniciados, que sin embargo, y gracias a su participación periférica y continuada, se ven recompensados con la incorporación gradual a una "cultura" específica. Así veremos que el alumno que práctica con Internet el acceso a fuentes documentales de Matemáticas, o de Estadística, atribuye a estas una naturaleza de verosimilitud y realidad — son recursos reales - difícilmente atribuibles a un texto o a un ejercicio ad hoc.

Objetivos, métodos, contenidos, condiciones de aprendizaje, evaluación y recursos constituyen las dimensiones comúnmente aceptadas del currículo. Es decir, el conjunto de intenciones acciones y decisiones que acompañan a cualquier proceso de intervención educativa o formativa $\mathrm{y}$, de forma previa, en la planificación curricular, durante las distintas evaluaciones de proceso, y después en la evaluación sumativa o final. El currículo puede ser más o menos implícito o explicito según se declaren o profundicen en mayor o menor grado las intenciones acciones y decisiones que lo 
constituyen y tendrá una distinta fundamentación según el eje del diseño y de su construcción se apoye en los principios que rigen la organización, los contenidos, los objetivos de formación, la situación de aprendizaje o los intereses de los alumnos etc. Pero en todo caso la idea central que rige los principios del diseño curricular es la de "pensar el currículo", es decir reflexionar individual o grupalmente el proceso sus condicionantes, desarrollo y conclusiones, como instrumento de trabajo y de comunicación.

\subsection{Conceptos y términos}

Aunque el término e-learning hay que utilizarlo, como después veremos, con reservas y cautelas para evitar confusiones conceptuales, lo cierto es que la práctica de los medios especializados -foros, listas de discusión, literatura especializada, etc.-, primero en Estados Unidos de Norteamérica y luego en el resto del mundo, ha acuñado este término para referirse en sentido amplio al mundo de la formación y de la educación que en algún momento o en la mayoría de los momentos utiliza las redes y los medios digitales como soporte de su actividad. También se ha acuñado este término en sentido más reducido para referirse a un mundo empresarial y a su correlativo en el mundo de la investigación tecnológica que ha tenido como objetivo distribuir conocimientos soportados en multimedia (multimedia educativo) o en la web con objeto de ser aprendidos por un sector más o menos preciso de destinatarios. Se trata de lo que comúnmente se conoce como el e-learning empresarial (Ruíperez, 2003) o del sector empresarial, del negocio en definitiva, que pone en circulación materiales de aprendizaje más o menos asistidos por un auténtico sistema de aprendizaje en redes. Este sector según lugares y circunstancias es un sector a mitad de camino entre el mundo editorial y el mundo procedente de las academias privadas y de la enseñanza por correspondencia, junto, en la Unión Europea, con un dinámico y pujante sector empresarial surgido al amparo de la política europea de programas de formación profesional y empresarial.

Este hecho ha conllevado a que algunos conceptos y términos se encuentren fuertemente concernidos e impregnados por matices procedentes de este mundo cuyos intereses son netamente comerciales y de costes económicos y afectados por temas derivados de su origen. Así al hablar de curso, se entiende de forma implícita algo muy parecido a un curso autosuficiente por correspondencia. Igual sucede al hablar de empaquetamiento, reusabilidad, etc. que incluso afecta al concepto en su totalidad, ya que al hablar de elearning, muchos de nosotros nos referimos a un sector económico, a un bussines. Nosotros para esto utilizaremos la expresión de e-learning empresarial. Mientras que elearning tecnológico lo utilizaremos para referirnos a su correlato en el mundo del desarrollo y de la producción informática, tanto en el sector de la industria como en el de la investigación.

A continuación realizaremos una breve digresión sobre algunos conceptos utilizados indistintamente en el e-learning empresarial y tecnológico con un sentido distinto al que de forma tradicional se utilizan en los ámbitos de las ciencias de la educación y del aprendizaje y, en particular, en los desarrollos propios del diseño curricular.

\section{Contenidos}

En el lenguaje que se utiliza en los ámbitos de teleformación empresarial y tecnológica se suele reservar este concepto para todas las informaciones datos y métodos que se 
almacenan, soportan y procesan en las plataformas y que no tienen relación estrictamente con lo que se utiliza para gestionar el sistema, dándole de forma implícita un carácter secundario o al menos equiparable al del hardware o al del software. De hecho para estas informaciones se utiliza el término courseware donde va implícita esta intención.

Bajo nuestra perspectiva, esta conceptualización se encuentra en el origen del fracaso de muchas iniciativas de formación apoyada en redes (sobre todo de las iniciativas de producción industrial de cursos y multimedia educativo), pues relativiza el carácter central de todo proceso de formación, la consecución de aprendizajes, a consideraciones de supuesta calidad tecnológica, brillantez en la resolución de procedimientos informáticos, programas y algoritmos, a consideraciones relacionadas en definitiva con el aspecto, con la presentación, con la resolución en los ambientes gráficos digitales, etc.

Utilizaremos el término contenido modificado por las expresiones de enseñanza o de aprendizaje ${ }^{2}$ con el sentido de material cognitivo que se ve aumentado o modificado en el aprendiz como resultado del proceso de aprendizaje. Los contenidos simplificando mucho pueden ser de distinto tipo: conceptuales, procedimentales o actitudinales.

Los datos e informaciones, no exclusivamente tecnológicos, que se soportan en las plataformas y en el web training, a los que de forma genérica se denominan materiales curriculares, por extensión de los términos utilizados en el diseño curricular no exclusivamente electrónico, está constituidos por materiales de distinto tipo, y no es objeto de este trabajo entrar en la discusión sino sólo situar al lector para entender lo que viene a continuación, y se clasifican en: guías didácticas, unidades didácticas, documentación (materiales donde se desarrollan los contenidos, son los clásicos apuntes, ejercicios y prácticas, documentos de apoyo, textos, imágenes, datos,... de carácter no específicamente formativo pero que se utilizan como apoyo o material de trabajo: Documentos profesionales o técnicos, tablas, documentos vivos, recursos de Internet...), multimedia y simulaciones de carácter formativo, guías de apoyo al alumno, instrumentos de evaluación de proceso, instrumentos de evaluación de aprendizaje, y documentación generada en la propia actividad de formación.

\section{Cursos}

En el e-learning empresarial se interpreta el término curso de forma clara como el material multimedia que se reúne en soporte digital o se deposita en una plataforma para desarrollar un programa de formación. En el caso extremo lo constituye un CD-ROM que se distribuye entre los usuarios, pero lo más frecuente es que se llame así a la unidad formativa que se constituye en una plataforma (Learning Management System) para que accedan de forma diferenciada los alumnos que siguen una propuesta formativa, a la que también se denomina curso.

De forma convencional ya sabemos que un curso es una unidad curricular que otorga acreditación o calificación propia, que eventualmente consta de módulos de aprendizaje

\footnotetext{
${ }^{2}$ Sustancialmente ambas expresiones — contenidos de enseñanza y contenidos de aprendizaje - quieren decir lo mismo, ya que lo que se quiere enseñar coincide con lo que se desea aprender, aunque enseñar y aprender sean actividades sustancialmente distintas.
} 
más pequeños: áreas, materias o bloques de contenidos, y que como cualquier otra unidad curricular en su configuración consta de las distintas dimensiones curriculares: objetivos, contenidos, metodología, evaluación, recursos, ...

\section{e-Learning}

Ya hemos dicho que la expresión e-learning se utiliza para denominar una actividad: la producción el soporte y la distribución de material multimedia de aprendizaje. Pero sin llegar a este extremo lo más frecuente es utilizarla como sustitutivo de formación apoyada en redes, sin distinguir lo que es aprendizaje de lo que es enseñanza (la actividad docente, organización de recursos educativos, etc.). En este sentido nosotros intentaremos proyectar los términos usuales en los entornos digitales, de esta forma distinguiremos e-learning, es decir lo que es propiamente aprendizaje (consecución de nuevos equilibrios cognitivos) en redes, de lo que es enseñanza en redes: $\boldsymbol{e}$-teaching. No obstante, como el aprendizaje en sistemas va indisolublemente asociado a la enseñanza utilizaremos expresiones como teleformación o sistemas de aprendizaje en redes para nombrar programas completos de intervención formativa o educativa que tengan como fin unos objetivos de esta naturaleza. Lo más frecuente es denominar en inglés estos programas utilizando el término training precedido de un modificante. Y así se habla de teacher training para nombrar a la formación docente o manager training para la formación en materia de organización.

\subsection{Objetos de aprendizaje}

Sin duda el mayor problema que aborda la industria del e-learning en la actualidad, aún sin resolver en aspectos muy fundamentales, es la ausencia de unas metodologías técnicas, documentales y psicopedagógicas comunes y aceptadas que garanticen los objetivos de accesibilidad, interoperabilidad, durabilidad y reutilización de los materiales curriculares basados en las redes.

En las actuales propuestas de formación en línea, lo que sucede generalmente es que los materiales preparados para un sistema no pueden ser transferidos a otro conservando sus características y propiedades.

La forma que se ha adoptado para resolver este problema es la de los estándares de learning: protocolos que contienen las especificaciones que permitirán dotar de flexibilidad a las propuestas de teleformación para su transferencia, tanto en el formato de los materiales y su estructura, así como en la conformación de las infraestructuras (herramientas informáticas y telemáticas).

Los estándares han iniciado el camino hacia una forma cómoda y viable de empaquetar los recursos y contenidos, tanto para los estudiantes que cambian de sistema, como para los docentes que utilizan en distintos contextos estos materiales, como para los desarrolladores que tienen que construir nuevas herramientas y mejorar las vigentes. Este proceso de convergencia de tecnologías es muy útil tanto a usuarios como para la industria del e-learning: A los productos que eventualmente se adhieran a estos estándares se les asegura que no quedarán obsoletos a corto o a medio plazo. De esta forma se protegen las inversiones realizadas.

La economía también se produce en el terreno del conocimiento. Así se prevé en los medios del e-learning empresarial. Los estándares comunes para los metadata de los 
materiales, el empaquetamiento y secuencia de los recursos, la interoperabilidad de herramientas, como las pruebas de conocimiento, los datos de rendimiento y los datos académicos de los alumnos, las interacciones con programas de aprendizaje en tiempo de ejecución, etc., ahorran inversión en aprendizajes adicionales y esfuerzos de diseño docente. Según estos medios son "requisitos indispensables para el éxito de la economía del conocimiento y para el futuro del e-learning". Sin embargo, como después señalaremos, este propósito se hace difícilmente compatible con los principios de la secuenciación de contenidos y con las leyes que rigen el entramado cognitivo que existe en los aprendices y permite la incorporación de conocimientos.

Varias han sido las iniciativası encaminadas a la estandarización de materiales de teleformación: AICC, Aviation Industry CBT Comitee; IEEE Learning Technologies Standards Comittee (LTSC); IMS Global Learning Consortium, Inc.; y ADLSCORM.

En noviembre de 1997 el gobierno de Estados Unidos puso en marcha una iniciativa encaminada a proveer herramientas, servicios y sistemas que permitiese llevar la formación y capacitación a todos los destinatarios, independientemente del lugar o la hora en que se encontrasen, pero sobre todo de la plataforma tecnológica que la instancia proveedora utilizase; de ahí surgió la iniciativa ADL (Advanced Distributed Learning).

La iniciativa ADL es un programa del Departamento de Defensa de los Estados Unidos y de la Oficina de Ciencia y Tecnología de la Casa Blanca para desarrollar principios y guías de trabajo para el desarrollo y la puesta en marcha eficiente, efectiva y en gran escala, de formación educativa basada en servicios y herramientas de la Web.

Esta iniciativa intenta recoger y recopilar "lo mejor" de las anteriores propuestas, refundiéndolas y mejorándolas en su propio estándar.

Así tres años después de ADL surge SCORM (Sharable Content Object Reference Model, Modelo de Referencia para Objetos de Contenidos Intercambiables), como resultado de esta organización; en este modelo se especificaron una serie de requisitos y pautas que definen cómo hacer para que el material que se utiliza en un sistema de aprendizaje basado en Internet sea transportado, integrado y reutilizado en distintas plataformas.

Surge pues una filosofía de compartir recursos de formación que de alguna forma está encaminada en uno u otro sentido a conformar y a afectar fuertemente el mundo de la formación a través de redes en la actualidad. De facto es la iniciativa que, por la vía de los hechos y de las declaraciones (aceptación por los fabricantes y declaraciones en los foros), se ha generalizado.

Algunos de los conceptos más importantes que se utilizan en esta filosofía son:

Empaquetamiento. Conjunto de especificaciones dirigidas a programadores y proveedores de materiales didácticos, LMS, sistemas de aprendizaje en redes y servicios formativos. Los objetos didácticos que se empaquetan contienen la descripción de la estructura y la localización de los materiales en línea, así como algunas características tecnológicas acerca de los datos contenidos.

Repositorios. Colecciones de recursos, documentos o informaciones de cualquier tipo accesibles a través de Internet o de una red digital. En el caso del e-learning pueden 
incluir los recursos de aprendizaje, los metadatos que describan dichos recursos o ambos.

Metadatos. Se trata de datos textuales estructurados. Conjunto estructurado de etiquetas descriptivas de objetos de información usadas para catalogar materiales educativos. Con ellos se trata de facilitar su localización y uso en la red o en un repositorio. Para ello incorporan los requisitos de los materiales y la descripción de la forma en que pueden ser implementados.

Diseño de aprendizaje. En el contexto de ADL-SCORN se debe entender como el desarrollo de un espacio de trabajo que permite conjugar la diversidad e innovación pedagógica con la posibilidad del intercambio de materiales interoperables y reutilizables. Su objetivo central es establecer criterios y métodos para el diseño instruccional de los materiales formativos utilizados.

El modelo SCORM está constituido, pues, por el conjunto de especificaciones que permiten desarrollar, empaquetar y distribuir materiales formativos en el lugar y momento precisos conservando sus funcionalidades y características.

Los materiales bajo estándar SCORM se elaboran asegurando del cumplimiento de cuatro principios:

1. Reusabilidad. Que los materiales curriculares y en particular los objetos de aprendizaje puedan ser reutilizados con diferentes herramientas, en distintas plataformas y en distintos contextos tecnológicos y curriculares. Y en qué grado se puede hacer.

2. Accesibilidad. Que las herramientas y productos que sigan estos estándares permitan un seguimiento del comportamiento y del historial de aprendizaje y académico de los alumnos

3. Interoperabilidad. Que se pueda intercambiar información a través de todas las plataformas (LMS.) que adopten los estándares

4. Durabilidad: Que los productos tecnológicos desarrollados con el estándar eviten la obsolescencia de los materiales.

Las pautas de SCORM, distribuidas por ADL, definen cómo deben publicarse los materiales y cómo deben usarse los metadatos. También incluyen las especificaciones para representar por medio de XML la estructura de los módulos de formación y cómo debe usarse el API (Application Programming Interface) ${ }^{3}$.

En general los criterios de SCORM se agrupan en tres categorías:

1. Los que se refieren a empaquetamiento de materiales. Tratan sobre la manera en que se guardan los contenidos de un módulo de formación (curso en la jerga de elearning industrial), el modo en que están vinculados entre sí y la forma en la que se entregará la información al usuario. Con referencia a un curso, estos datos se concentran en un archivo llamado imanifest.xml

\footnotetext{
${ }^{3}$ API: Aplication Program Interface. Literalmente interfaz para programas de aplicación. Conjunto de convenios de programación que establecen cómo se invoca un servicio desde un programa.
} 
2. Los que se refieren a la ejecución de las comunicaciones. Detallan el ambiente para ejecutar la información y se agrupan en dos partes: los comandos de ejecución y los metadatos del estudiante.

3. Los metadatos del curso. Son de dos tipos: los que incluyen la información del curso propiamente y los que se refieren al material del estudiante.

La versión actualizada de SCORM es la 1.3, que puede obtenerse en la dirección http://www.adlnet.org/.

Un ejemplo de buena práctica de SCORM puede encontrarse igualmente en www.scorm.tamucc.edu/.

\subsection{Objetos de aprendizaje reutilizables}

La filosofía de compartir recursos va más allá de los cursos en línea. Así los objetos de aprendizaje reutilizables son recursos digitales que pueden integrarse en distintos contextos curriculares apoyando programas formativos con distintos objetivos, destinatarios, etc. y que pueden reutilizarse indistintamente sin adaptación.

De esta forma L'Allier (1997) los define como "la mínima estructura independiente que contiene un objetivo, una actividad de aprendizaje y un mecanismo de evaluación" y Wiley (2002) los describe como "cualquier recurso digital que se puede utilizar como apoyo para el aprendizaje".

Como vemos el concepto resultante de unir ambas definiciones es amplísimo y se puede aplicar prácticamente a cualquier objeto didáctico en soporte digital con enormes diferencias de importancia en la intervención formativa y de niveles de uso, desde una presentación en una clase presencial, o una fotografía digital para explicar, por ejemplo, en una clase de arte una ventana gótica, hasta una asignatura completa virtualizada.

Un concepto diferente es el de reusabilidad. Como veremos un objeto no es absolutamente reutilizable en distintos contextos tecnológicos o curriculares. En nuestro caso nos centraremos en esto último y en los problemas que suscita en el campo del diseño instruccional. En este sentido, la cuestión básica que se plantea es en qué grado un objeto es reutizable.

Por lo tanto, se puede argumentar que la reusabilidad (Sicilia, Miguel-Angel y García, Elena, 2003) es la característica esencial más importante de los objetos de aprendizaje. Sin embargo, puesto que la reusabilidad se refiere a situaciones anticipadas y usos futuros, es difícil de medir. Esto exige que la especificación de los contextos posibles de uso determine el grado de reusabilidad del objeto de aprendizaje y que la reusabilidad total se pueda definir como el grado resultante de suficiencia para cada uno de los contextos posibles especificados.

La reusabilidad no es única sino que depende del contexto. Por lo tanto, no es simple ni unidimensional. La reusabilidad de un objeto de aprendizaje también debe ir referida a las distintas caracteristicas que lo definen y así hablaremos por ejemplo de secuenciabilidad.

Se acepta comúnmente que el diseño de los objetos de aprendizaje implica básicamente tres disciplinas: diseño instruccional, informática y bibliotecnología. 
El diseño instruccional, tal como se entiende a través de ADL-SCORM, permite definir los objetivos educativos que rigen la creación de los objetos de aprendizaje. La informática, la telemática,... las tecnologías digitales en definitiva, como es obvio, constituyen la base operativa desde la que se construyen este tipo de recursos; apoyándose para ello en la filosofía de la programación orientada a objetos, poniendo especial énfasis en aspectos como compartir, heredar e integrar recursos para atender diferentes objetivos.

Finalmente, la bibliotecnología y las ciencias de la documentación proveen métodos y teorías de catalogación para el acceso, la clasificación, el almacenamiento y la búsqueda de recursos.

No obstante como señalamos al principio el objetivo central de los objetos de aprendizaje consiste en alcanzar la posibilidad de que los alumnos y los docentes puedan adaptar los recursos formativos en concordancia con sus objetivos de formación y de aprendizaje, intereses, necesidades y estilos de aprendizaje y de enseñanza.

Tal como lo entienden los teóricos de los objetos de aprendizaje reutilizables, el reto al que se enfrentan las empresas y centros de formación e investigación que se dedican al e-learning empresarial, y posiblemente otros centros que lo utilicen, con relación a los propios objetos de aprendizaje, a los repositorios que los almacenarán y a las herramientas que los procesan, es no solamente brindar la posibilidad de encontrar contenidos de aprendizaje (entiéndase programas formativos), sino contextos significativos y relevantes, para los estudiantes, donde ubicar los contenidos elaborados (Longmire, 2002).

\section{2}

\subsection{Contenidos y secuenciación}

La organización y la secuenciación de los contenidos de enseñanza constituyen el punto neurálgico del itinerario que nos conducirá a diseñar los procesos de aprendizaje.

En otro momento (Esteban, M. y Zapata, M., 1992) nos hemos referido a la concreción y contextualización de las intenciones formativas, con un triple propósito que la orientación de las acciones e intervenciones formativas que realizamos:

- responda las necesidades específicas de los alumnos en un determinado contexto,

- sea coherente con las opciones que confieren carácter propio a la institución que las organiza o al programa formativo, o al centro,

- en su caso incluya los elementos preceptivos del currículo establecido por la Administración para el programa formativo.

Ya vimos (Esteban, M. y Zapata, M., 1992) que esto tiene su proyección en la selección y organización de los contenidos, es decir que la concreción de los objetivos educativos orientará los procesos de enseñanza-aprendizaje y repercutirá también en los otros aspectos de la planificación del currículo (del Proyecto Curricular), sobre todo en la selección y en el enfoque de los contenidos de enseñanza y en la evaluación del proceso de aprendizaje. Esto parece obvio, es implícito a cualquier proceso de planificación curricular y tiene su correlación en las correspondientes intervenciones formativas. 
Por eso, es preciso señalar que los diversos componentes del Currículo (fundamentos, objetivos, contenidos, evaluación y recursos), que habitualmente se tratan por separado, están interrelacionados. De manera que en la práctica tanto de la planificación como en la propia intervención formativa y en la evaluación tendremos que ir revisando de forma recurrente lo que vamos haciendo, los resultados obtenidos, compararlos con los objetivos previstos y efectuar las mejoras convenientes. Tanto es así, que a medida que avancemos en el estudio de cada uno de ellos tendremos que echar la vista atrás para enriquecer y perfilar las formulaciones hechas previamente. Y esto afecta también a la selección y a la secuenciación de contenidos. Como veremos la mayor parte de las veces estos procesos están incluidos en las técnicas que describimos a continuación.

Por último cabe decir que las consideraciones incluidas en este trabajo tienen una relación muy estrecha, o son las mismas, que las que se pueden utilizar para la selección, organización y distribución de los contenidos de enseñanza por ciclos más amplios que los que corresponden a un módulo formativo, o a cualquiera de las unidades curriculares con las que se trabaje en un programa de los que habitualmente impartimos. Lo que sucede es que, por razones fáciles de comprender, esta tarea corresponde a otras instancias y a otros niveles de decisión.

\subsection{Presupuestos básicos de la secuenciación de contenidos.}

Plantear la cuestión de la organización y secuenciación de los contenidos de enseñanza implica tener presentes unos cuantos presupuestos básicos que determinarán muchas de nuestras opciones. Recordémoslo rápidamente.

Primero. Los objetivos generales establecidos para cada nivel de planificación, acordados previamente, tendrán que incidir en la acción formativa para cada nivel inferior de planificación a través de los objetivos generales establecidos para ese nivel y para los contenidos de ese nivel.

De esta forma, por ejemplo, los objetivos generales establecidos para un programa formativo (master, especialización,...) acordados previamente tendrán que incidir en la acción educativa a través de los objetivos generales de los distintos cursos y en los contenidos establecidos para el curso, de igual forma estos objetivos, los objetivos generales del curso, incidirán en los objetivos generales de las distintas materias o áreas del currículo y de los contenidos de enseñanza (conceptuales, procedimentales y actitudinales).

La progresión en los contenidos por áreas, cursos, programas o niveles formativos no se realiza exclusivamente, como frecuentemente se suele aceptar, de forma lineal como si dependiera de una sola variable: la cantidad de contenidos, a repartir ésta en distintas unidades de tiempo, o de su naturaleza, sino obedeciendo a multiplicidad de criterios.

Así la progresión puede obedecer sólo a criterios de secuenciación propia de los contenidos (progresión lineal, en primero los números naturales, en segundo los enteros, en tercero los racionales y en cuarto los reales), o de la naturaleza de estos (por áreas de conocimiento: en primero el álgebra, en segundo la geometría, etc.), o pueden seguir otros modelos o sistemas de avance (progresión en espiral, recurrente, basado en resolución de problemas, etc.) o cualquier otro eje globalizador. O incluso puede seguir criterios derivados de los propios contenidos, teniendo en cuenta criterios de planificación curricular. Es decir atendiendo a los objetivos generales que se desglosan 
en objetivos parciales y que implican que el alumno incorpora los contenidos correspondientes.

Por tanto, el diseño de los procesos de aprendizaje en cada una de las unidades, áreas, módulos o niveles, tendrá que incluir tanto los contenidos de enseñanza como los objetivos educativos que se pretenden alcanzar en esa unidad, área, módulo o nivel.

Además, a riesgo de ser reiterativos, no nos debe importar recordar con frecuencia que la secuenciación de los contenidos de enseñanza tiene que referirse a los tres tipos de contenidos:

- hechos, conceptos y principios;

- procedimientos y algoritmos, y

- actitudes, valores y normas.

Segundo. En el alumno, y en un caso óptimo, se van a desarrollar las capacidades y se van a incorporar los contenidos de aprendizaje establecidos para el nivel módulo o materia correspondiente, pero también se van a desarrollar otras capacidades o conocimientos no previstos, o en la correspondiente etapa de desarrollo personal se deben desarrollar otros conocimientos que corresponden a esa etapa. Por tanto los objetivos generales de la unidad o nivel que estamos trabajando, vinculados a contenidos particulares de los tres tipos indicados, han de orientarse a la formación integral de todos los alumnos.

Por tanto, al programar el trabajo y al seleccionar y secuenciar los contenidos, tenemos que referirnos a la adquisición de las capacidades cognitivas, psicomotrices, de equilibrio y de autonomía personal, de relación interpersonal y de inserción social, en el marco de los objetivos de la unidad correspondiente.

En efecto, el aprendizaje de los contenidos ha de considerarse como una aportación al pleno desarrollo de la personalidad del alumno. Por eso, los contenidos de enseñanza y los objetivos educativos tienen que abarcar más aspectos formativos que los que podrían derivarse de los planteamientos específicos que estamos considerando.

Tercero. La aceptación de los principios del aprendizaje significativo comporta concebir los procesos de enseñanza-aprendizaje desde la óptica de la construcción del conocimiento por parte del alumno.

Como veremos, este presupuesto incidirá de manera particular en la orientación de las decisiones referentes a los criterios que determinarán la secuenciación de los contenidos de enseñanza, la selección de las estrategias de aprendizaje y la elección de los materiales curriculares que convendrá poner a disposición de los alumnos.

Cuarto. Nuestra concepción de la formación exige, como imperativo ético, que diseñemos procesos de enseñanza-aprendizaje adecuadamente diferenciados, con el fin de poder atender a la diversidad de capacidades e intereses de los alumnos.

En efecto, una formación que favorece la atención a la diversidad tiene que asegurar a todos los alumnos la adquisición de los contenidos básicos y promover al máximo el desarrollo de cada uno de ellos sin ningún tipo de discriminación. 
Este presupuesto implica diferenciar adecuadamente lo que es básico e indispensable en el aprendizaje, de lo que es resultado de ampliación o de profundización y, por ello, sólo estará al alcance de algunos alumnos; además, las jerarquías de aprendizaje tendrán que contemplar la diversidad de puntos de partida de los alumnos y las modalidades específicas de acceder a la adquisición de los diversos tipos de contenidos.

Antes de iniciar la tarea de secuenciación de los contenidos de enseñanza convendrá que los equipos de profesores nos pongamos de acuerdo respecto a estos presupuestos básicos.

Si todos compartimos los mismos criterios respecto a los cuatro puntos enunciados, el trabajo en equipo será más fácil y eficaz.

\subsection{Finalidad de la secuenciación de los contenidos de enseñanza.}

La finalidad de la secuenciación es establecer una ordenación de los contenidos de enseñanza que asegure el enlace entre los objetivos educativos y las actividades de aprendizaje de los alumnos, de tal manera que la organización del trabajo formativo dé garantías suficientes para la consecución de las intenciones formativas propias del programa de formación, la comunidad educativa o de la institución.

Damos por supuesto que los contenidos de enseñanza de un área determinada son interdependientes y que el orden en que son propuestos a los alumnos no es indiferente para el aprendizaje.

Trataremos tres técnicas de secuenciar los contenidos: La basada en el análisis de los contenidos, la basada en el análisis de la tarea y la teoría de la elaboración.

El intento de combinar las dos técnicas primeras ha dado lugar la teoría de la elaboración, muy recomendada en la bibliografía relativa a la reforma como pauta para la secuenciación del aprendizaje.

No obstante, antes de trazar de los criterios de secuenciación inherentes a la teoría de la elaboración interesa analizar por separado y valorar críticamente las aportaciones específicas de cada una de las dos técnicas citadas, con el fin de destacar algunos elementos que pueden sernos útiles en la secuenciación de los contenidos de enseñanza.

\subsection{La técnica de análisis de contenidos.}

En el estadio más evolucionado, el análisis de contenidos proporciona criterios de secuenciación que tienen en cuenta tanto la estructura interna de los contenidos de enseñanza como los procesos cognitivos que intervienen en el aprendizaje significativo.

Nadie pone en duda que el conocimiento de las estructuras internas del conocimiento ya elaborado facilita su comprensión y retención y favorece la continuidad de la enseñanza. Sin embargo, la estructura lógica del contenido, considerada como un punto de partida para la secuenciación de las actividades de aprendizaje, no es necesariamente lo mejor para facilitar los aprendizajes de los alumnos.

Y esto por una razón: no podemos confundir la estructura formal interna de un conjunto de conocimientos y la estructura que conviene dar a este conjunto de conocimientos para que los alumnos puedan aprenderlo con relativa facilidad. 
Por ello, no será suficiente tener en cuenta las características de los contenidos del área o materia que hay que enseñar a los alumnos, sino que habrá que partir de la situación en que se encuentran respecto al aprendizaje de estos contenidos y a la forma en que construirán sus conocimientos.

Teniendo en cuenta estas consideraciones, según la técnica de análisis de contenidos el proceso a seguir para secuenciar un conjunto de contenidos de enseñanza consta de tres pasos:

1. Descubrir y destacar los ejes vertebradores de los contenidos que deben enseñarse a los alumnos.

2. Descubrir y destacar los contenidos fundamentales y organizarlos en un esquema jerárquico y relacional.

\section{Proceder a la secuenciación según los principios de la organización psicológica del conocimiento.}

Según J.D. Novak, los principios que rigen la organización psicológica del conocimiento pueden resumirse de la manera siguiente:

1. Todos los alumnos pueden aprender significativamente un contenido a condición de que dispongan de conceptos relevantes e inclusores en su estructura cognoscitiva.

2. El contenido del aprendizaje debe ordenarse de tal manera que los conceptos generales e inclusivos -por lo tanto, los más importantes- se presenten al principio. Esto favorece la formación de conceptos inclusores en la estructura cognoscitiva de los alumnos que facilitan, posteriormente, el aprendizaje significativo de los otros elementos del contenido.

3. Las secuencias de aprendizaje tienen que ordenarse partiendo de los conceptos más generales y avanzando de forma progresiva hacia los conceptos más específicos, con el fin de lograr no sólo una diferenciación progresiva del conocimiento del alumno - es decir, la incorporación a su estructura cognoscitiva de nuevos elementos que enriquecen y diversifican los inclusores iniciales-, sino también una reconciliación integradora posterior - es decir, la coherencia del conjunto de conceptos de la estructura cognoscitiva.

4. Después de presentar los conceptos más generales e inclusivos del contenido, la introducción de los elementos posteriores debe hacerse mostrando tanto las relaciones que mantienen con los primeros como las relaciones que mantienen entre sí.

Esta manera de proceder facilita la diferenciación progresiva y la reconciliación integradora.

5. La presentación inicial de los conceptos más importantes, generales e inclusivos del contenido debe apoyarse en ejemplos concretos que los ilustren empíricamente.

A modo de síntesis cabe afirmar que la secuenciación de contenidos de enseñanza debe hacerse teniendo en cuenta tres criterios generales que orientarán la organización de los bloques de contenido para el proceso de aprendizaje de los alumnos: 
Primer criterio: La elaboración de secuencias de aprendizaje por parte de los profesores supone considerar la estructura del contenido de enseñanza que hay que proponer a los alumnos y, a la vez, la manera como los alumnos construyen su propio conocimiento.

Segundo criterio: Los contenidos seleccionados como fundamentales deben ser los que tienen mayor capacidad de inclusión, es decir, los que pueden integrar otros contenidos que los alumnos también tendrán que aprender; y cuantos más contenidos puedan integrar, mejor.

Tercer criterio: En primer lugar hay que presentar los conceptos más generales e inclusivos, dejando para después los aspectos más concretos y los más irrelevantes.

Con estos criterios, siguiendo los tres pasos indicados más arriba, el análisis del contenido de enseñanza conduce al establecimiento de unas jerarquías conceptuales que suponen una secuenciación descendente: comienzan por los contenidos más generales e inclusivos hasta llegar a los más específicos, pasando por contenidos intermedios.

Para la elaboración de secuencias de aprendizaje, en particular las que se refieren a contenidos de carácter conceptual, pueden ser útiles los llamados mapas de conceptos, los esquemas conceptuales, las representaciones en forma de árbol, los diagramas de Venn, etc.

Los defectos que podrían derivarse de la aparente rigidez de una secuenciación de este tipo se pueden evitar presentando de forma cíclica los diversos contenidos, con la finalidad de provocar la diferenciación progresiva y la reconciliación integradora, poniendo de relieve las relaciones existentes entre ellos.

Se observará que este enfoque es perfectamente compatible con una interpretación constructivista del aprendizaje y de la intervención pedagógica, y que los principios enunciados pueden aplicarse indistintamente a bloques de contenidos más o menos amplios, de manera que son igualmente válidos en los diferentes momentos de concreción previstos en el diseño curricular.

Con todo, nuestra reflexión se ha centrado prioritariamente, y casi exclusivamente, en los contenidos de carácter conceptual. Y esto porque los criterios válidos para la secuenciación de contenidos se refieren sólo a las relaciones entre conceptos. Por otra parte, es bien sabido que no es posible reducir a conceptos todos los contenidos de enseñanza y sólo intentarlo supondría un grave atentado a nuestro compromiso de promover la educación integral de los alumnos. 




\subsection{La técnica de análisis de tareas.}

El análisis de tareas, es decir, la determinación y descripción de las actividades (componentes de ejecución que conducen a la adquisición de una destreza), es una técnica que permite secuenciar los contenidos en términos de resultados esperados del aprendizaje de los alumnos.

En este enfoque se da por supuesto que el dominio de las habilidades intelectuales de nivel inferior implica procesos de aprendizaje más elementales que el dominio de las habilidades de nivel superior. La consecuencia es obvia: cuando una tarea compleja se puede descomponer en tareas más elementales o sencillas, que se corresponden a capacidades o destrezas de orden inferior, hay que empezar por realizar tareas más sencillas y acabar por las más complejas.

En este marco de referencia los contenidos de enseñanza se definen en términos de objetivos de ejecución, que especifican lo que el alumno tiene que ser capaz de hacer en relación a los contenidos que aprende. Así, para cada bloque de contenidos será preciso determinar un conjunto de tareas (objetivos de ejecución), y la realización de estas tareas comportará la adquisición y el dominio de los contenidos correspondientes.

Según este criterio, el objetivo terminal (criterio de evaluación) previsto determinará las habilidades intelectuales que son necesarias para alcanzarlo. A su vez, estas habilidades determinarán aquellas otras más sencillas que habrá que aprender ordenadamente, empezando por las que están en el nivel inferior de la jerarquía establecida; es decir, las que responden a la capacidad inicial del alumno y que, por tanto, puede realizar sin demasiado esfuerzo.

Según la técnica de análisis de tareas, el proceso a seguir para secuenciar los contenidos de enseñanza comporta tres pasos:

1. Determinar la tarea que el alumno debe realizar (habilidad que tiene que aprender).

2. Determinar los posibles componentes de la tarea o habilidad (subtareas o subhabilidades). 
3. Secuenciar las subtareas o subhabilidades, de la más sencilla a la más compleja.

En los estadios más evolucionados, el análisis de tareas no se limita a especificar los objetivos de ejecución y determinar la secuencia de actividades que deben realizarse, sino que trata de identificar los procesos y las estructuras psicológicas que acompañan a la realización de las diferentes actividades. Es decir, intenta poner de relieve la competencia específica correspondiente a la realización de una tarea concreta.

Por tanto, el análisis de tareas intenta descubrir y explicar cómo operan las personas (qué procesos realizan) con los datos adquiridos previamente (información) para resolver una determinada tarea (ejecución).

Desde este punto de vista, la secuenciación de contenidos de enseñanza comporta:

- descubrir cómo debe estructurarse la información para facilitar la ejecución de la tarea prevista;

- determinar las estrategias cognitivas y los procedimientos que deben aplicarse para la ejecución de la tarea, que equivaldrá a la adquisición de la habilidad deseada y al aprendizaje del contenido correspondiente.

La técnica de análisis de tareas no está suficientemente desarrollada para garantizar una correcta y adecuada secuenciación de todos los contenidos de enseñanza y el correspondiente proceso de aprendizaje de los alumnos, pero es útil para secuenciar algunos procedimientos que persiguen el desarrollo de determinadas habilidades o destrezas (intelectuales, de manipulación, de comportamiento, etc.).

En efecto, hay contenidos de enseñanza que se prestan a ser traducidos en tareas de ejecución, pero con otros es prácticamente imposible. Por eso, sería muy arriesgado "convertir" todo un bloque de contenidos (conceptos, procedimientos, valores...) en tareas que los alumnos tienen que realizar. Las deficiencias del resultado serían evidentes.

Por tanto, la secuenciación realizada aplicando únicamente la técnica de análisis de tareas basada en jerarquías de aprendizaje corre un doble peligro: por un lado, dejar aspectos importantes del contenido de enseñanza que no pueden traducirse en tareas de ejecución; y, por el otro, no considerar intenciones educativas que requieren aprendizajes de mayor complejidad.

\subsection{La teoría de la Elaboración.}

La teoría de la elaboración integra elementos de la técnica de análisis de contenidos y de la técnica de análisis de tareas, en un esquema coherente que pretende superar sus deficiencias. El resultado es la propuesta de las secuencias elaborativas como una forma de secuenciar los contenidos de enseñanza.

La secuencia elaborativa se fundamenta en el principio siguiente:

Los contenidos de enseñanza tienen que ordenarse de manera que los elementos más simples y generales ocupen el primer lugar, incorporando después, de manera progresiva, los elementos más complejos y detallados. 
Según este principio en un primer momento convendrá presentar una panorámica global de las partes principales de que constan los contenidos de enseñanza, pasando luego a la elaboración de cada parte por separado y volviendo de vez en cuando a la visión de conjunto con la intención de enriquecerla y ampliarla. De este modo, una vez elaborada una parte de la panorámica inicial, en una primera fase de análisis, se vuelve al punto de partida con el fin de situar en la visión de conjunto lo que se ha elaborado, y se procede de forma análoga con las diferentes partes del contenido global hasta que todas se hayan elaborado en un primer nivel de complejidad.

El proceso se puede repetir tantas veces como sea necesario hasta que se alcance el nivel de detalle deseado. Con ello se pretende que los alumnos puedan abordar los contenidos de enseñanza en el nivel de complejidad más apropiado al estado de sus conocimientos respectivos.

En resumen, la teoría de la elaboración postula que los resultados del aprendizaje serán mejores, cualitativa y cuantitativamente, en la medida en que la organización de la enseñanza se aproxime a un modelo que:

- presente el contenido que es objeto de enseñanza en términos muy generales y simples, a modo de panorámica global;

- introduzca el nivel de complejidad deseado en cada uno de los componentes de esta panorámica global, y procure incorporar en ella las elaboraciones sucesivas.

Al final de cada uno de los niveles de elaboración las secuencias elaborativas requieren un resumen y una síntesis: el resumen incluye una revisión de los elementos de contenido que se han incorporado en el correspondiente nivel de elaboración; y la síntesis muestra las relaciones que estos elementos mantienen entre sí y con aquellos otros de los que no son más que un desarrollo.

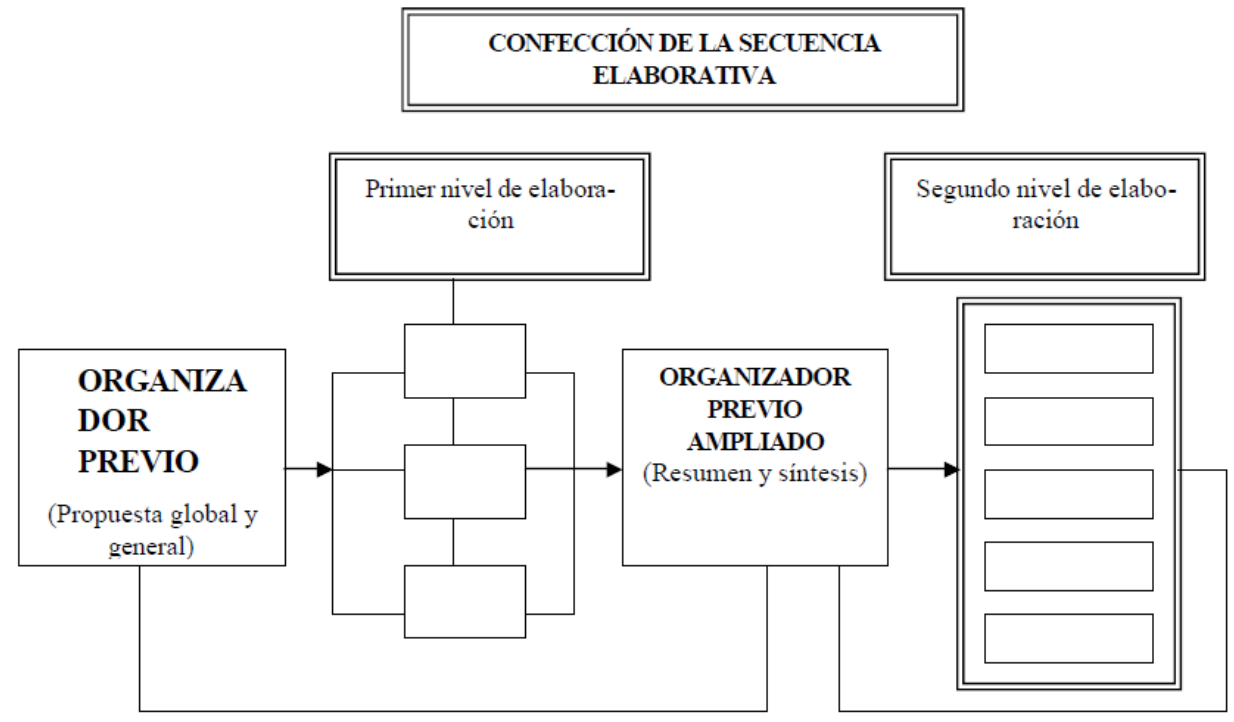




\section{El organizador previo en la secuencia elaborativa.}

La panorámica global que constituye el primer paso de la secuencia elaborativa recibe el nombre de organizador previo o epítome.

Los organizadores previos se consideran un medio muy eficaz para potenciar la estructura cognitiva del alumno, ya que favorecen la retención de las nuevas informaciones y ayudan a interrelacionar adecuadamente los conocimientos previos de los alumnos con los elementos fundamentales del nuevo contenido de enseñanza.

Por tanto, los organizadores previos han de servir de puente entre los conceptos relevantes ya presentes en la estructura cognitiva del alumno y los contenidos que se le quieren enseñar. Si estos conceptos relevantes no existiesen, el organizador previo serviría para construir un concepto inclusor que facilitara el nuevo aprendizaje.

Los organizadores previos más eficaces son los que utilizan conceptos, términos y proposiciones con los que el alumno ya está familiarizado y que pueden presentarse fácilmente por medio de ejemplos y analogías. El oganizador previo tiene estas propiedades:

- no incluye todos los elementos importantes del contenido solo los aportes más importantes y representativos;

- sus elementos se eligen de manera que el resto del contenido aporte más detalle o mayor complejidad (la secuencia irá de lo más general a lo más detallado, y de lo más simple a lo más complejo);

- el organizador previo es objeto de enseñanza;

- el organizador previo tendrá una aplicación práctica, por el medio de ejemplos, ejercicios o ilustraciones empíricas, con la finalidad de hacerlo significativo para el alumno;

- el organizador previo y la secuencia elaborativa partirán de un determinado contenido que actuará como hilo conductor prioritario a lo largo de la secuencia, y este contenido podrá ser de tipo conceptual, procedimental o actitudinal;

- los elementos del contenido que no responden a la orientación elegida como prioritaria se introducirán en el momento adecuado en función de la relevancia que tengan para el desarrollo de todo el proceso de aprendizaje.

\section{La confección del organizador previo.}

Según la teoría de la elaboración, la confección de un organizador previo para la secuenciación de contenidos de enseñanza comporta tres pasos.

1. Escoger el contenido de aprendizaje que podrá servir como organizador previo.

2. Seleccionar los elementos más fundamentales y representativos del contenido escogido.

3. Seleccionar otros elementos relevantes para la enseñanza del organizador previo. 
Los criterios prácticos sugeridos por la teoría de la elaboración para confeccionar secuencias de contenidos de enseñanza tienen una conexión evidente con los principios del aprendizaje significativo, pero no son suficientes, por sí mismos, para orientar todas las decisiones referentes a la secuenciación, ni se pueden aplicar de la misma manera a los diferentes tipos de contenidos de enseñanza. Las operaciones que implica el proceso de secuenciación de contenidos de cada una de las áreas del currículo son complejas, los problemas a resolver en cada una de las áreas del currículo también y los problemas a resolver en cada caso no siempre tienen fácil solución.

Por tanto, la teoría de la elaboración puede considerarse válida en la medida en que nos orienta en la realización de una tarea necesaria, pero no podemos esperar que dé solución inmediata a toda la problemática inherente al proceso de secuenciación de contenidos de enseñanza. En cualquier caso, es compatible con los planteamientos indicados en los apartados anteriores.

\section{Conclusiones}

Simplificando mucho podemos decir que en el mundo de la teleformación hay dos polos de tensión: el que llamaremos de recursos abiertos y el industrial. El primero viene representado por la iniciativa MIT's OpenCourseWare $(\mathrm{OCW})^{4}$ del Massachusetts Institute of Technology (MIT) ${ }^{5}$ y el segundo por los complejos de la industria del elearning y la Secretaría de Defensa de los Estados Unidos ${ }^{6}$.

En el primer caso se trata de compartir a través de la simplicidad, los recursos abiertos y la ética del hacker $^{7}$ (Himanen, Pekka. 2002), poniendo el énfasis no en el carácter completo pero cerrado de los recursos sino en la metodología, en la intervención tutorial y evaluativo, en definitiva en la atención personal y sobre todo en la superación de los objetivos de aprendizaje (adquisición de conceptos y de destrezas y habilidades así como en la ejecución de procedimientos). En el segundo caso también se pone el énfasis en la consecución de objetivos de aprendizaje pero en aras de una mayor eficiencia en términos de costes se pone el énfasis en la autosuficiencia de las herramientas y recursos (plataformas, multimedias, etc).

Los sistemas más autosuficientes implican unos formatos de datos para los recursos curriculares tecnológicamente mucho más complejos, donde se tiene que atender a una gran variedad de funciones pedagógicas automatizadas o semiatumatizadas. Por no hablar de funcionalidades organizativas o a mitad de camino entre lo organizativo y lo pedagógico, y sobre todo donde la industria del e-learning y la investigación tecnológica tiene más campo de actuación, más diente que clavar.

\footnotetext{
${ }^{4}$ http://ocw.mit.edu/index.html

${ }^{5} \mathrm{http} / / /$ web.mit.edu/index.html

${ }^{6} \mathrm{http} / / /$ www.adlnet.org/index.cfm?fuseaction=adlhist. En la página de corporaciones que adoptan SCORM http://www.adlnet.org/index.cfm?fuseaction=adoptersearch se puede encontrar una relación completa de empresas de e-learning que utilizan este estándar.

${ }^{7}$ Este sistema de ideas éticas que tiene su origen en las actitudes de los primitivos hackers — programadores altruistas y apasionados que programaban y ofrecían sus trabajos de forma abierta y gratuita - tiene su definición y estudio más acabado en la obra de Pekka Himanen La ética del hacker y el espíritu de la era de la información.

Se trata de una nueva moral que contradice la ética calvinista del trabajo, tal como la definió Max Weber. La ética protestante y capitalista está basada en la laboriosidad eficiente, la aceptación del orden y la rutina, el valor del dinero y la preocupación por los resultados en términos de beneficios dinerarios. Frente a esto, la nueva ética del trabajo se fundamenta en el valor de la creatividad en el trabajo y en compartir los recursos.

El beneficio deja de medirse por el dinero. Y se fija en índices como el valor social de lo producido, el libre acceso (open access), la transparencia y la franqueza en las relaciones interpersonales.
} 
A medida que aumenta la riqueza en la automatización de las funciones o funcionalidades educativas, docentes, etc. aumenta por un lado la necesidad de contar con entornos cuya amistosidad supere o palie la rudeza o lentitud de los entornos operativos (informáticos) y las dificultades de comunicación o de relación entre los usuarios. Y por otro, añadir operaciones a los recursos implica una mayor complejidad a la hora de diseñar los estándares de intercambio, transportabilidad e interoperacionalidad y, por lo tanto, se hace cada vez más difícil encontrar formatos estándares. Esto de alguna manera supone caer en una espiral de complejidades.

Dejamos pues de lado esta tensión entre sistemas abiertos y sistemas industriales. Los primeros no presentan problemas de transporte, integración y reutilización ya que con utilizar formatos estándares de datos y de procedimientos informáticos (PDF para los documentos y HTML, XML o Java Sript para multimedia o animaciones) se soluciona el problema.

En el segundo caso se añade una nueva tensión a la ya descrita, compatibilizar el criterio de calidad según el cual un formato es mejor cuantas más funcionalidades docentes y cognitivas incorpore con el criterio de compatibilidad tecnológica: transportabilidad, integrabilidad y reusabilidad. Esta tensión, inherente al propio concepto de e-learning empresarial, es la que define el polo hacia donde deben orientarse la actividad y el esfuerzo desarrollador e investigador.

Por último como conclusiones intentaremos abordar tan sólo en un nivel general, simplemente como enunciados, varias cuestiones. La primera de ellas es una cuestión básica y previa: ¿el concepto de objeto de aprendizaje reutilizable es compatible con los requisitos de interdependencia de contenidos de aprendizaje? Y en todo caso ¿qué requisitos se tendrían que asegurar para ello? A continuación nos tendremos que plantear de forma necesaria una serie de cuestiones:

¿Cómo se pueden o se deben trasladar los criterios de secuenciación a los objetos de aprendizaje?, ¿cómo se deben trasladar en la fase de diseño y de elaboración?, ¿cómo deben operar en la fase de ejecución?, ¿qué datos, informaciones y criterios se añadan a las informaciones de otro tipo que se adjuntan a los objetos de aprendizaje?, ¿se hace como apoyo a la intervención educativa o de forma automatizada?.

Por otra parte habrá que tener en cuenta si la eficiencia de un sistema de este tipo complica excesivamente el artificio tecnológico a que pueda dar lugar, o bien si éste enturbia los aprendizajes, en qué medida lo hace y si merece la pena ¿Puede dar la tecnología respuesta a cuestiones tan complejas como ésas con una casuística tan matizada e imprevisible de antemano, o más bien entra dentro del dominio del conocimiento experto sólo atendible desde la intervención tutorial personal no automatizada?

Para abordar estos temas los ilustraremos con algunos ejemplos. Trataremos primero las cuestiones generales y a continuación nos limitaremos a ejemplos sólo en el caso primero, la técnica de secuenciación mediante el análisis de contenidos.

\subsection{Cuestiones generales}

Recordemos que la finalidad de la secuenciación es establecer una ordenación de los contenidos de enseñanza que asegure el enlace entre los objetivos educativos y las 
actividades de aprendizaje de los alumnos, de tal manera que la organización de la actividad desarrollada dé garantías suficientes para la consecución de las intenciones formativas propias del programa de formación, la comunidad educativa o de la institución.

Para decirlo de una forma sencilla: La secuenciación de contenidos, de tareas y de actividades ha de ir propiciando un acercamiento progresivo desde la situación inicial de aprendizaje de los alumnos hasta los objetivos propuestos para el programa formativo de que se trate por la comunidad educativa o por la institución. Y esto es distinto para cada colectivo de alumnos destinatarios de la formación e incluso para cada individuo, para cada programa formativo y para cada institución. Por tanto la información adecuada que se suministra debe ser explícita y debe estar en correspondencia con cada una de las situaciones en las que se produce el proceso. También tendrá que ser objeto de estudio no solo cómo se organiza la información sino también como se operativiza en caso de que sea posible o cómo se apoya, si se realiza mediante una tutorización personal, a través de los individuos y mediada por los ordenadores, o bien de forma automatizada mediante herramientas informáticas.

\begin{tabular}{|l|l|}
\hline $\begin{array}{l}\text { Criterio } \\
\text { La secuenciación de contenidos, de } \\
\text { tareas y de actividades }\end{array}$ & $\begin{array}{l}\text { Variables: } \\
\text { La secuenciación debe ir adaptada al } \\
\text { menos }\end{array}$ \\
\hline $\begin{array}{l}\text { ha de ir propiciando un acercamiento } \\
\text { progresivo desde la situación inicial de } \\
\text { aprendizaje de los alumnos hasta los } \\
\text { objetivos propuestos para el programa } \\
\text { formativo. }\end{array}$ & $-\begin{array}{l}\text { para cada colectivo de alumnos destinatario } \\
\text { de la formación, }\end{array}$ \\
\hline
\end{tabular}

Por otra parte otro supuesto básico es que los contenidos de enseñanza de un área determinada son interdependientes y que el orde $n$ en que son propuestos a los alumnos no es indiferente para el aprendizaje.

Un mismo objeto de aprendizaje colocado en un contexto, dentro de un orden y para ser utilizado en un momento determinado, contará con una situación de conceptos y procedimientos previos desarrollados, en esa o en otras áreas, que será distinto de cualquier otra. La situación de aprendizaje de los alumnos también será en general distinta en cuanto al andamiaje cognitivo: organizadores previos, conceptos inclusores, ideas implícitas etc. para contextos formativos, grupos de destinatarios e incluso para individuos distintos.

Cuando introducimos un objeto de aprendizaje o una unidad de contenidos (un concepto, un procedimiento,...) debemos asegurar que se cumple con carácter general este principio.

Como tal principio general no es operativo en sí mismo, no va a inspirar una regla o un procedimiento válido en todos los casos, pero sí debe inspirar los procedimientos de contextualización del objeto de aprendizaje. Y seguramente llegaremos a la conclusión de que esto no es posible en el cien por cien de los casos, de manera que muy probablemente se dé lugar a conceptos redundantes o a situaciones contradictorias, que choquen no ya con los principios del aprendizaje constructivo, y den lugar a conflictos 
cognitivos, sino que incluso puedan chocar con las reglas de la lógica (lo definido no debe entrar en la definición, no utilizar como apoyo conceptos posteriores para definir conceptos anteriores, etc.). La cuestión que se planteará entonces es cómo seguir, si automatizar los procedimientos o adaptar los entornos para estas funciones.

\begin{tabular}{|l|l|l|}
\hline $\begin{array}{l}\text { Los contenidos de } \\
\text { enseñanza }\end{array}$ & Los objetos de aprendizaje \\
\hline $\begin{array}{l}\text { de un área determinada son } \\
\text { interdependientes }\end{array}$ & $\begin{array}{l}\text { Un mismo objeto de aprendizaje colocado en un contexto, } \\
\text { centro de una secuencia, y para ser utilizado en un momento } \\
\text { determinado, contará con una situación de conceptos y } \\
\text { procedimientos previos desarrollados en esa o en otras áreas } \\
\text { que será distinto de cualquier otra situación. }\end{array}$ \\
\hline $\begin{array}{l}\text { el orden en que son propuestos a } \\
\text { para el aprendizaje }\end{array}$ & $\begin{array}{l}\text { cambian en función del lugar que ocupen en la secuencia. } \\
\text { La situación de aprendizaje de los alumnos también será en } \\
\text { general distinta en cuanto al andamiaje cognitivo: } \\
\text { organizadores previos, conceptos inclusores, ideas implícitas } \\
\text { etc. para contextos formativos, grupos de destinatarios e } \\
\text { incluso para individuos distintos. }\end{array}$ \\
\hline
\end{tabular}

A un nivel más particular cualquiera de las técnicas consideradas para la secuenciación de contenidos tiene implicaciones claras no solo para el diseño instruccional de los objetos de aprendizaje sino sobre todo para la propia construcción de los objetos.

Un estudio más detallado nos llevaría a un análisis de estas implicaciones con relación al menos a las tres técnicas descritas, sin embargo no es el objetivo de este trabajo profundizar en ese tema sino simplemente enunciar la cuestión llamando la atención sobre esta necesidad. Por tanto nos vamos a limitar a hacer unos comentarios sobre la técnica de análisis de contenidos.

Según la técnica de análisis de contenidos el proceso a seguir para secuenciar un conjunto de contenidos de enseñanza consta de tres pasos: descubrir y destacar los ejes vertebradores de los contenidos que deben enseñarse a los alumnos; descubrir y destacar los contenidos fundamentales y organizarlos en un esquema jerárquico y relacional; y por último proceder a la secuenciación según los principios de la organización psicológica del conocimiento. En el apartado correspondiente hemos señalado cuales eran según Novak y Gowin (Novak y Gowin, 1984; Novak, 1990), los principios que rigen la organización psicológica del conocimiento y hemos hecho un resumen de ellos.

De ello al menos se concluye como ejemplo para el procedimiento general que: 


\begin{tabular}{|l|l|}
\hline Fase del procedimiento & Implica (criterio de reusabilidad) \\
\hline $\begin{array}{l}\text { Descubrir y destacar los ejes } \\
\text { vertebradores de los contenidos que } \\
\text { deben enseñarse a los alumnos }\end{array}$ & $\begin{array}{l}\text { Que los objetos de aprendizaje en la información que se acompaña y en } \\
\text { su diseño instruccional (actividades propuestas, referencias, } \\
\text { ejemplificaciones, etc.) se tenga en cuenta el eje que vertebra el } \\
\text { programa de formación donde va incluido. Y si no es así que se haga un } \\
\text { tratamiento genérico que evite la particularizaciones, de manera que si es } \\
\text { útil para un curso o materia no lo sea para otro: Se haga con ello } \\
\text { irreutilizable. } \\
\text { Por ejemplo, no es lo mismo tratar el tema de derivadas en matemáticas } \\
\text { para la representación de funciones, para resolver problemas de máximos } \\
\text { y mínimos, o para ajustar curvas por el método de mínimos cuadrados. }\end{array}$ \\
\hline $\begin{array}{l}\text { Descubrir y destacar los contenidos } \\
\text { fundamentales y organizarlos en un } \\
\text { esquema jerárquico y relacional }\end{array}$ & $\begin{array}{l}\text { Que el objeto vaya provisto de un esquema (mapa) de contenidos } \\
\text { organizados de forma jerarquizada según el eje vertebrador elegido. }\end{array}$ \\
\hline
\end{tabular}

Igualmente se puede considerar las implicaciones que tiene para el diseño de OAR los principios que rigen la organización psicológica del conocimiento:

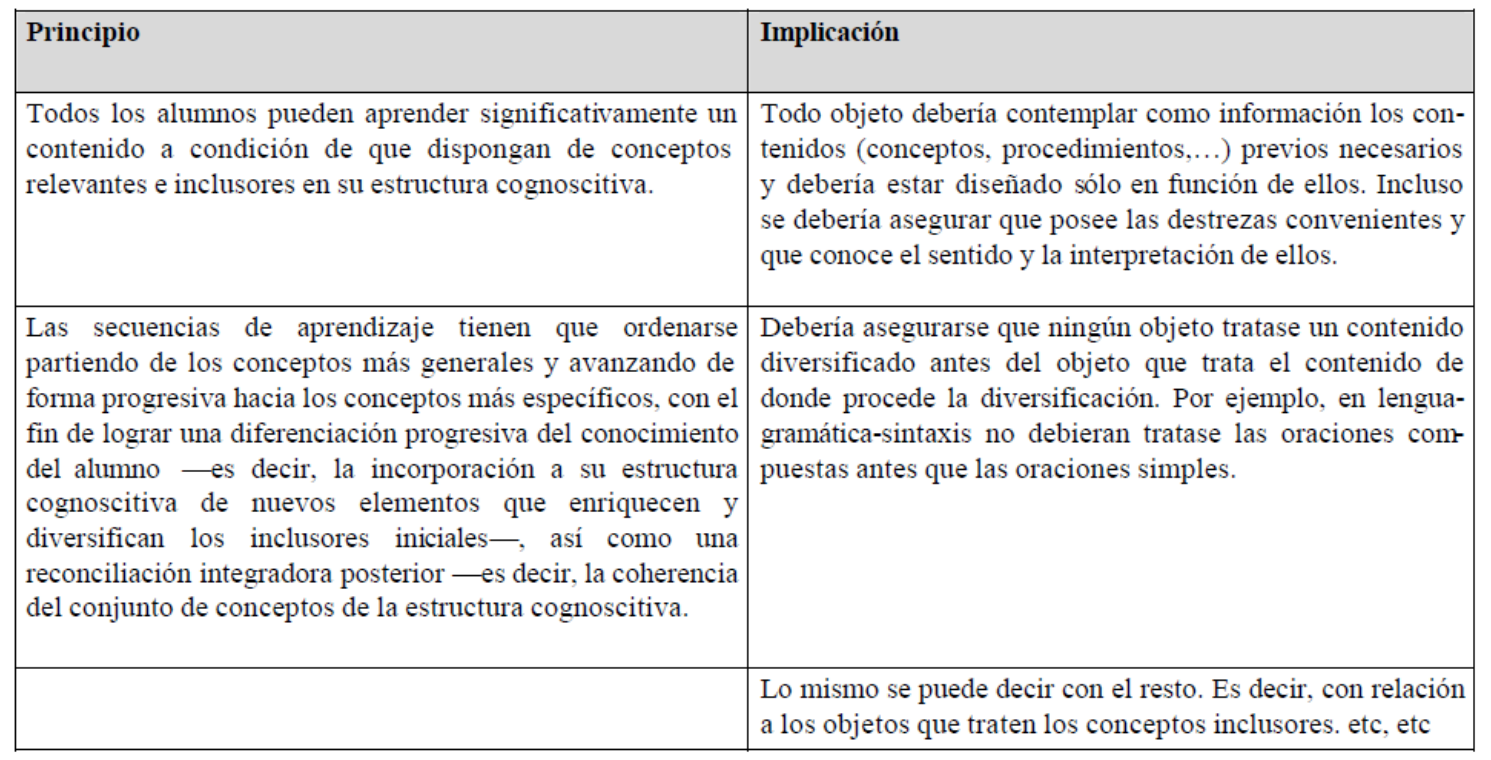

Con estos criterios, siguiendo el análisis del contenido de enseñanza, se llega finalmente a unos requisitos operativos que permiten definir la secuenciabilidad de los objetos de aprendizaje reutilizables. Naturalmente hay que insistir en que el proceso es complejo puesto que la secuencia de los contenidos no se corresponde necesariamente con la "secuencia lógica o natural de los contenidos" y que la secuenciabilidad podría tener como origen criterios distintos de análisis sobre cómo se forman los conceptos y las ideas (análisis de contenidos) o bien pueden proceder del análisis de la tarea o de la teoría de la elaboración. E incluso podría concluirse que hay objetos de aprendizaje no secuenciabilizables.

En cualquier caso creemos que esta reflexión, este análisis y este esfuerzo nos acercarán a hacer los contenidos más asumibles por los alumnos y por añadidura les permitirán hacer una mejor apropiación de ellos. 
Concluido en Murcia a 3 de Febrero de 2005

Zapata, M. (2016). Secuenciación de contenidos y objetos de aprendizaje. RED. Revista de Educación a Distancia, 50. Consultado el (dd/mm/aaaa) en http://www.um.es/ead/red/50

\section{Referencias}

Antunez, S. et al.: Del Projecte Educatiu a la programació d'aula. Graó Editorial, Barcelona, 1991.

Ausubel, D. P.: Psicología educativa: un punto de vista cognitivo. Trillas, México, 1976.

Ausubel, D .P.; novak, J .D.; Hanesian, H.: Psicología de la educación. Trillas, México, 1983.

Beltran, J.: Aprender a aprendender. Estrategias cognitivas. Cincel, Madrid, 1989.

Briggs, L.: La ordenación de secuencias en la instrucción. Guadalupe, Buenos Aires, 1973.

Brown, John Seely; Collins, Allan; and Duguid, Paul. Situated Cognition and the Culture of Learning. Educational Researcher, V18, N1, págs. 32-42, Jan/Feb 1989.

Bruner, J.S.: Hacia una teoría de la instrucción. Uthea, México, 1969.

Bruner, J.S.: El proceso de la educación. Uthea, México, 1972.

Bruner, J.S.: Investigaciones sobre el desarrollo cognitivo. Pablo del Río, Madrid, 1980.

Bruner, J.S.: Desarrollo cognitivo y educación. Morata, Madrid, 1988.

Cole, M.: “Conclussion”. En L. Resnick, J. Levine y S. Teasley (eds.) Perspectives on socially shared cognition. Washington, D.C., Americam Psychological Association, 1991.

Coll, C.: Psicología y currículum. Laia, Barcelona, 1989.

Coll, C.: Aprendizaje escolar y construcción del conocimiento. Paidós, Barcelona, 1990. Cuadernos de Pedagogía, números 139, 168, 170, 172, 174, 175, 177, 178, 180193, Fontalba S.A., Barcelona. 
Crook, Ch.: Computers and the collaborative experience of learning. Routledge -11 New Fetter Lane, London EC4P 4EE, 1994

Crook, Ch. (1998): Ordenadores y aprendizaje colaborativo. Ediciones Morata, SL. Madrid. Departamente d'ensenyament de la Generalitat de Catalunya: Disseny Curricular. Ensenyament Secundari Postobligatori. Batxillerat. Publicaciones del Departament d'Ensenyament. Barcelona, 1990.

Eisner, E.: Procesos cognitivos y currículo. Martínez Roca, Barcelona, 1987.

Esteban, M y Zapata, M.: "Aprendizajes y recursos tecnológicos. Documentación para la formación del profesorado del Proyecto Atenea". CEP de Murcia, 1992.

Gagné, E. D.: "La psicología cognitiva del aprendizaje escolar". Aprendizaje-Visor. Madrid, 1991.

Gagné, R .M.; Briggs, L. J.: La planificación de la enseñanza. Trillas, México, 1976 (7. a edición 1986).

Gagné, R. M.: Las condiciones del aprendizaje. Aguilar, Madrid, 1971.

Gimeno, J.: El currículo: una reflexión sobre la práctica. Morata, Madrid, 1988.

Himanen, Pekka: La ética del hacker y el espíritu de la era de la información. Ed. Imago Mundi. Prólogo de Linus Torvalds. Epílogo de Manuel Castells, 2002. http://www.edestino.es/etica_hacker.htm.

L'Allier, James J.: Frame of Reference: NETg's Map to the Products, Their Structure and Core Beliefs. NetG, 1997.

http://www.netg.com/research/whitepapers/frameref.asp y http://www.netg.com/research/frameref.htm

Longmire, Warren: "Content and Context: Designing and Developing Learning Objects" Learning Without Limits. Vol. 3. Infomania, 2000.

Longmire, Warren: A primer on learning objects, 2002. www.learningcircuits.org/mar2000/primer.html

Novak, J.D.; Gowin, D.B.: Aprendiendo a aprender. Martínez Roca, Barcelona, 1977.

Novak, J.D.: Teoría y práctica de la educación. Alianza Universidad, Madrid, 1988.

Novak, J.D. y Gowin, D.B.: Learning how to learn. Cambridge, MA: Cambridge University Press, 1984.

Novak, J.D.: Concept maps and Vee diagrams: two metacognitive tools to facilitate meaningfud learning. Instructional Science, 19, 29-52, 1990. 
Papert, S.; Mindstorms: Children, Computers, and Powerful Ideas, Basic Books, New York, 1980.

Papert, S: Desafío a la mente. Computadoras y educación. Buenos Aires: Galápago, 1981.

Papert, S.: The Children's Machine: Rethinking School in the Age of the Computer. New York: Basic Books, 1993.

Papert, S.: The Connected Family: Bridging the Digital Generation Gap. Longstreet Press, 1996.

Papert, S.: La familia conectada. Emecé, Abril 1997

Papert, S.: Tomorrow's Classrooms? Times Educational Supplement March 5, 1982 (pp. 31-32, 41) http://www.papert.org/articles/TomorrowsClassrooms.html.

Pask, G.: Conversation, cognition and learning. Amsterdam and New York: Elsevier, 1975.

Porlán, R.; García, J.E.; Cañal, P.: Constructivismo y enseñanza de las ciencias. Diada Editoras, Sevilla, 1988.

Pozo, J. J.: Teorías cognitivas del aprendizaje. Morata, Madrid, 1990.

Reggini, H.: Alas para la mente. Galápagos Marzo 1983

Reggini, H.: Ideas y formas. Buenos Aires: Galápago, 1985.

Rodríguez-Roselló, L.: Logo. De la tortuga a la inteligencia artificial. Madrid: Vector, 1986.

Rodríguez-Roselló, L.: Logo: Un lenguaje de ordenador para la enseñanza. En J. Laborda (Ed.), Informática y Educación. Barcelona: Laia, 57-74, 1986.

Rodríguez-Roselló, L.: Material de formación del profesorado del Proyecto Atenea. MEC-PNTIC. Madrid, 1988.

Ruíperez, G.: Educación virtual y eLearning. Biblioteca. Fundación AUNA. Madrid. (p.105), 2003

S.E.C.C.: El currículo escolar: qué es, qué pretende, de qué consta y cómo se aplica. Publicaciones del Secretariado de la Escuela Cristiana, Barcelona. 1989.

S.E.C.C.: La LOGSE: lo que dice y lo que no dice. Publicaciones del Secretariado de la Escuela Cristiana, Barcelona, 1990.

S.E.C.C.: La LOGSE: qué pretende y cómo incidirá en la acción educativa. Publicaciones del Secretariado de la Escuela Cristiana, Barcelona, 1990. 
Shayer, M.; Adey, P.: Enseñanza y aprendizaje de las Ciencias. Narcea, Madrid, 1984.

Sicilia, M. A. y García, E.: On the Concepts of Usability and Reusability of Learning Objects. International Review of Research in Open and Distance Learning (October - 2003). http://www.irrodl.org/content/v4.2/sicilia-garcia.html 19-Sep-04; 17:06:55.

Stenhouse, L.: Investigación y desarrollo del currículo. Morata, Madrid, 1984.

Vigotsky, L.S.: El desarrollo de los procesos cognitivos superiores. Crítica, Barcelona, 1984.

Wiley, D. A.: "Connecting Learning Objects to Instructional Design Theory: A Definition, a Metaphor, and a Taxonomy." The Instructional Use of Learning Objects. Bloomington, IN: Agency for Instructional Technology, 2002.

Young, M. F.: Instructional design for situated learning. Educational Technology Research \& Development, 41(1), 43-58, 1993.

Zabalza, M. A.: Diseño y desarrollo curricular. Narcea, Madrid, 1987.

Zapata, M.: Evaluación de un Sistema de Gestión del Aprendizaje. RED. Revista de Educación a Distancia, núm. 9. Departamento de Psicología Evolutiva y de la Educación de la Universidad de Murcia, 2003. http://www.um.es/ead/red/9 http://www.um.es/ead/red/9/eval_SGA_1.pdf

\footnotetext{
${ }^{\mathrm{i}}$ Referencias sobre estándares de e-learning:

La información sobre distintos sistemas de estándares se puede completar a partir de las siguientes referencias:

1. e-learning Standards and Technical Specifications, Mats Svensson, 26 Nov. 2001 (www.luvit.com)

2. Making Sense of Learning Specifications \& Standards: A Decision Maker's Guide to their Adoption, The MASIE Center e-Learning CONSORTIUM, 8 Marzo 2002.

3. e-learning interoperability Standards, Sun Microsystem Inc.

4. Utilización de SCORM en el diseño de cursos y sistemas de gestión de aprendizaje en entorno Web, Begoña Perela Moreta.

5. Click2learn Briefing: e-learning Standards, Claude Ostyn, Learning System Strategist, Click2learn Inc., Noviembre 2001.

6. A standard for success, P.J. Connolly, Octubre 2001. URL: http://www.infoworld.com/articles/tc/xml/01/10/15/011015tclearn.xml

7. e-learning: Standars, Paul Stacey, Marzo 2001, URL: http://www.bctechnology.com/statics/pstaceymar0201.html

8. CETIS Standards compliant products directory, Mayo 2002, URL: http://www.cetis.ac.uk/directory

9. QS Media, e-learning, URL: http://www.qsmedia.es/elearning/default.cfm

10. SCORM Concepts, URL: http://www.eduworks.com/LOTT/tutorial/scormconcepts.html

11. IEEE Learning Technology Standards Committee (LTSC), URL: http://ltsc.ieee.org/

12. IMS (Instructional Management System) Global Learning Consortium, URL: http://www.imsproject.org/

13. Advanced Distributed Learning (ADL) Initiative, URL: http://www.adlnet.org

14. AICC, The Aviation Industry CBT Committee, URL: http://www.aicc.org/
} 